Article

\title{
Base-Promoted Chemodivergent Formation of 1,4-Benzoxazepin-5(4H)-ones and 1,3-Benzoxazin-4(4H)-ones Switched by Solvents
}

\author{
Qian Chen, Yunpeng Wang and Ruimao Hua * \\ Department of Chemistry, Tsinghua University, Key Laboratory of Organic Optoelectronics \& Molecular \\ Engineering of Ministry of Education, Beijing 100084, China; chenqian9482@126.com (Q.C.); \\ wangyp16@mails.tsinghua.edu.cn (Y.W.) \\ * Correspondence: ruimao@mail.tsinghua.edu.cn; Tel.: +86-10-6279-2596
}

Academic Editor: Alejandro Baeza Carratalá

Received: 12 September 2019; Accepted: 18 October 2019; Published: 19 October 2019

\begin{abstract}
The $\mathrm{KOH}$-promoted chemodivergent benzannulation of ortho-fluorobenzamides with 2-propyn-1-ol can afford either 1,4-benzoxazepin-5(4H)-ones or 1,3-benzoxazin-4(4H)-ones in good yields with high selectivity, depending greatly upon the use of solvents. In the case of using DMSO, the intermolecular benzannulation produced seven-membered benzo-fused heterocycles of 1,4-benzoxazepin-5(4H)-ones, whereas in $\mathrm{MeCN}$, the six-membered benzo-fused heterocycles of 1,3-benzoxazin-4(4H)-ones were formed. The $\mathrm{KOH}$-promoted benzannulation proceeded most probably through the $\mathrm{C}-\mathrm{F}$ nucleophilic substitution of ortho-fluorobenzamides with 2-propyn-1-ol to give the intermediate of ortho-[(2-propynyl)oxy]benzamide, which underwent the intramolecular hydroamidation in a different manner to afford either seven- or six-membered benzo-fused heterocycles.
\end{abstract}

Keywords: base-promoted; chemodivergent formation; solvent-dependent transformation; 1,4-benzoxazepinones; 1,3-benzoxazinones

\section{Introduction}

Chemodivergent reactions are interesting and efficient protocols that form the structurally different heterocyclic compounds from the same starting materials through simple change of reaction conditions [1-10]. Among them, the solvent-dependent or solvent-controlled chemodivergent reactions have been well applied in the synthesis of heterocyclic compounds [11-21]. On the other hand, benzo-fused seven- and six-membered heterocycles containing two heteroatoms of oxygen and nitrogen such as 1,4-benzoxazepin-5(4H)-ones [22], 3,4-dihydro-1,4-benzoxazepin-5(2H)-ones [23,24], 2,3,4,5-tetrahydro-1,4-benzoxazepines [25-29], and 2,3-dihydro-1,3-benzoxazin-4(4H)-ones [30-32], are important and interesting heterocyclic compounds due to their wide spectrum of biological activities (Scheme 1). In addition, as the structures shown in Scheme 1, 1,4-benzoxazepin-5(4H)-one is the useful and potential precursor for the synthesis of its derivatives by simple transformation. Therefore, the synthetic approach to 1,4-benzoxazepin-5(4H)-one ring has been well investigated. However, the known procedures for the formation of the seven-membered benzo-fused heterocycle either are multi-step with low atom-utilization or using uneasily available starting materials catalyzed by palladium complexes [33-35].

As part of our continued interest in the development of the application of base/DMSO-promoted $\mathrm{S}_{\mathrm{N}} \mathrm{Ar}$ reaction for the formation of $\mathrm{C}-\mathrm{N}$ bond under transition-metal-free conditions [36-38], and the chemodivergent transformations of alkynes [39-41], as well as the new synthetic methods of heterocyclic compounds [42-44], we herein describe an efficient protocol for the 
formation of 1,4-benzoxazepin-5(4H)-ones and 2-vinyl-1,3-benzoxazin-4(4H)-ones via KOH-promoted solvent-controlled intermolecular cyclization reaction between substituted ortho-fluorobenzamide and 2-propyn-1-ol [45].

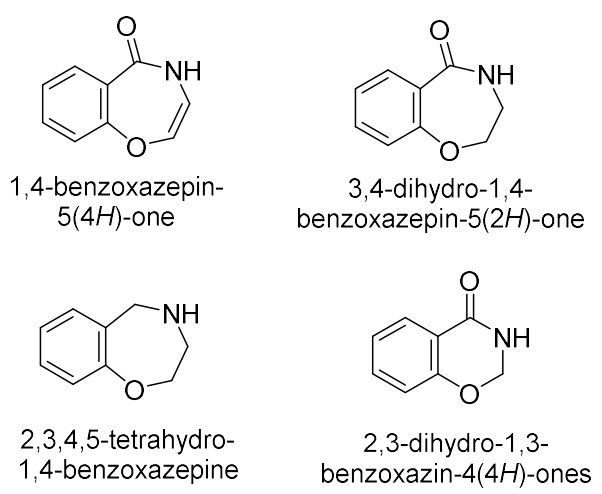

Scheme 1. 1,4-Benzoxazepin-5(4H)-ones, 1,3-benzoxazin-4(4H)-ones, and their derivatives.

\section{Results and Discussion}

Table 1 concludes the results from the reaction of ortho-fluoro- $N$-propylbenzamide (1a) with 2-propyn-1-ol. We firstly examined the reaction of 1 a with 2-propyn-1-ol (1.2 equivalents) in the presence of $\mathrm{KOH}$ (3.0 equivalents) in DMSO at $50{ }^{\circ} \mathrm{C}$ for $12 \mathrm{~h}$. Two products could be isolated from the reaction mixture, and their structures were confirmed to be $N$-propyl-3-methyl-1,4- benzoxazepin-5(4H)-one (2a, 45\%) and $N$-propyl-2-vinyl-2,3-dihydro-1,3-benzoxazin-4(4H)-ones (3a, 12\%) (entry 1) [46]. When the same reaction was repeated at $30{ }^{\circ} \mathrm{C}$, the yield of $2 \mathrm{a}$ was slightly increased (entry 2 ), and if the reaction was carried out at $30^{\circ} \mathrm{C}$ for $12 \mathrm{~h}$ firstly and then $50{ }^{\circ} \mathrm{C}$ for $12 \mathrm{~h}, 2 \mathrm{a}$ was isolated in $54 \%$ yield (entry 3). In this case, 3a was determined only in a small amount $(<5 \%)$ in the reaction mixture with the complete conversion of $\mathbf{1 a}$. The use of either 1.0 equivalent or 1.5 equivalents of 2-propyn-1-ol resulted in the decrease of $2 \mathbf{a}$ yield (entries 4 and 5). Increase of the reaction temperature to $70{ }^{\circ} \mathrm{C}$ for $12 \mathrm{~h}$ also led to the lower yield of $\mathbf{2 a}$ (entry 6). Decreasing the amount of $\mathrm{KOH}$ to 1.0 or 2.0 equivalents affected the formation of $2 \mathrm{a}$ (entries 7 and 8). Other inorganic bases, such as $\mathrm{NaOH}, \mathrm{K}_{2} \mathrm{CO}_{3}, \mathrm{Cs}_{2} \mathrm{CO}_{3}$, and $t$-BuOK were not efficient in promoting the cyclocondensation (entries 9-12). Very interestingly, the screening of solvents disclosed that the selective chemodivergent formation of $2 \mathbf{a}$ and $3 \mathbf{a}$ greatly depended on the use of solvents (entries 13-18). In the cases of THF, DMF and a mixture solvent of $\mathrm{DMSO} / \mathrm{MeCN}$ used, 3a was the major product, and in $\mathrm{MeCN}$, 3a could be isolated in $83 \%$ yield with a small amount of $\mathbf{2 a}$ formation. In addition, either increasing the amount of 2-propyn-1-ol or increasing the reaction temperature did not improve the yield of 3a (entries 19 and 20).

The cyclocondensation of other substrates $\mathbf{1} \mathbf{b}-\mathbf{k}$, bearing different substituents on nitrogen or benzene ring with 2-propyn-1-ol, was then studied under the conditions of entry 3 in Table 1 . As can be seen from Scheme 2, N-alkyl-2-fluorobenzamides (alkyl = Me (1b), isopropyl (1c), $t$-butyl (1d), t-amyl (1e), cyclopentyl (1f), cyclohexyl (1g)) showed similar reactivity to 1a affording the

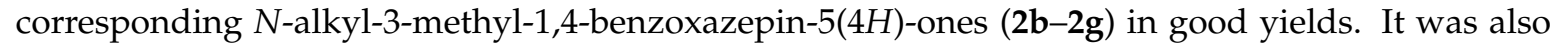
noted that $N$-phenyl-2-fluorobenzamide showed unexpectedly sluggish reactivity, and almost all starting materials could be recovered after the reaction under the same conditions. In addition, the results from the reactions of 2-fluoro-3-methyl-N-propylbenzamide (1h), 2-fluoro-4-methoxy- $N$ propylbenzamide (1i), and 4-chloro-2-fluoro- $N$-propylbenzamide (1j) with 2-propyn-1-ol apparently indicated that the electron-withdrawing group in $\mathbf{1 j}$ was unfavorable in the formation of 1,4-benzoxazepin-5(4H)-one ring. Moreover, the reaction of 2-fluorobenzamide (1k), bearing an unprotected $\mathrm{NH}_{2}$ group, afforded the corresponding product (2k) in only $31 \%$ yield. It should be noted that the reaction of ortho-fluorobenzamides bearing a strong electron-withdrawing group, such as 2-fluoro-5-nitro- $N$-propylbenzamide (11) and 2-fluoro- $N$-propyl-5-trifluoromethyl- benzamide (1m), resulted in a complex mixture. 
Table 1. Effects of reaction conditions on the chemodivergent formation of 1,4-benzoxazepin-5(4H)-ones and 1,3-benzoxazin-4(4H)-ones ${ }^{a}$.

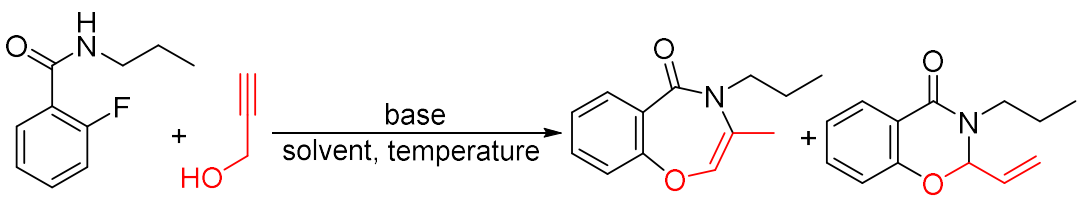
1a 1.2 equivalents
$3 a$

\begin{tabular}{|c|c|c|c|c|}
\hline Entry & Base (equivalent) & Solvent & Temperature $\left({ }^{\circ} \mathrm{C}\right) /$ Time $(\mathrm{h})$ & Yield (\%) ${ }^{b}$ \\
\hline 1 & $\mathrm{KOH}(3)$ & DMSO & $50 / 12$ & $45 \%(2 \mathbf{a})+12 \%(3 \mathbf{a})$ \\
\hline 2 & $\mathrm{KOH}(3)$ & DMSO & $30 / 12$ & $52 \%(2 \mathbf{a})+7 \%$ \\
\hline 3 & $\mathrm{KOH}(3)$ & DMSO & $30 / 12+50 / 12$ & $54 \%(2 \mathbf{a})$ \\
\hline $4^{c}$ & $\mathrm{KOH}(3)$ & DMSO & $30 / 12+50 / 12$ & $45 \%(2 \mathbf{a})$ \\
\hline $5^{d}$ & $\mathrm{KOH}(3)$ & DMSO & $30 / 12+50 / 12$ & $47 \%(2 a)$ \\
\hline 6 & $\mathrm{KOH}(3)$ & DMSO & $30 / 12+70 / 12$ & $41 \%(2 a)$ \\
\hline 7 & $\mathrm{KOH}(1)$ & DMSO & $30 / 12+50 / 12$ & $44 \%(2 a)$ \\
\hline 8 & $\mathrm{KOH}(2)$ & DMSO & $30 / 12+50 / 12$ & $47 \%(\mathbf{2 a})$ \\
\hline 9 & $\mathrm{NaOH}(3)$ & DMSO & $30 / 12+50 / 12$ & $33 \%(2 a)$ \\
\hline 10 & $\mathrm{~K}_{2} \mathrm{CO}_{3}(3)$ & DMSO & $30 / 12+50 / 12$ & $\sim 10 \%(2 \mathbf{a})$ \\
\hline 11 & $\mathrm{Cs}_{2} \mathrm{CO}_{3}(3)$ & DMSO & $30 / 12+50 / 12$ & comlex mixture \\
\hline 12 & $t$-BuOK (3) & DMSO & $30 / 12+50 / 12$ & complex mixture \\
\hline $13^{e}$ & $\mathrm{KOH}(3)$ & DMAc & $30 / 12+50 / 12$ & $52 \%(2 a)+26 \%(3 a)$ \\
\hline 14 & $\mathrm{KOH}(3)$ & 1,4-dioxane & $30 / 12+50 / 12$ & $35 \%(2 \mathbf{a})+\sim 10 \%(3 \mathbf{a})$ \\
\hline 15 & $\mathrm{KOH}(3)$ & THF & $30 / 12+50 / 12$ & $\sim 10 \%(2 a)+48 \%(3 a)$ \\
\hline 16 & $\mathrm{KOH}(3)$ & DMF & $30 / 12+50 / 12$ & $\sim 10 \%(2 a)+48 \%(3 a)$ \\
\hline 17 & $\mathrm{KOH}(3)$ & $\mathrm{MeCN}$ & $30 / 12+50 / 12$ & trace $(2 \mathbf{a})+83 \%(3 \mathbf{a})$ \\
\hline 18 & $\mathrm{KOH}(3)$ & $\mathrm{MeCN}$ & $30 / 12+50 / 12$ & $12 \%(2 \mathbf{a})+53 \%(3 \mathbf{a})$ \\
\hline $19^{d}$ & $\mathrm{KOH}(3)$ & DMSO/MeCN (1:1 in volume) & $30 / 12+50 / 12$ & $\operatorname{trace}(\mathbf{2 a})+79 \%(3 \mathbf{a})$ \\
\hline 20 & $\mathrm{KOH}(3)$ & $\mathrm{MeCN}$ & $30 / 12+70 / 12$ & $\operatorname{trace}(2 \mathbf{a})+62 \%(3 \mathbf{a})$ \\
\hline
\end{tabular}

${ }^{a}$ Unless otherwise noted, the reactions were carried out using $0.5 \mathrm{mmol}$ of $1 \mathrm{a}, 0.6 \mathrm{mmol}$ of 2-propyn-1-ol, and $1.5 \mathrm{mmol}$ of base in $4.0 \mathrm{~mL}$ of solvent. ${ }^{b}$ Isolated yields. ${ }^{c} 0.5 \mathrm{mmol}$ of 2-propyn-1-ol was used. ${ }^{d} 0.75 \mathrm{mmol}$ of 2-propyn-1-ol was used. ${ }^{e}$ DMAc: $N, N$-dimethyl acetamide.

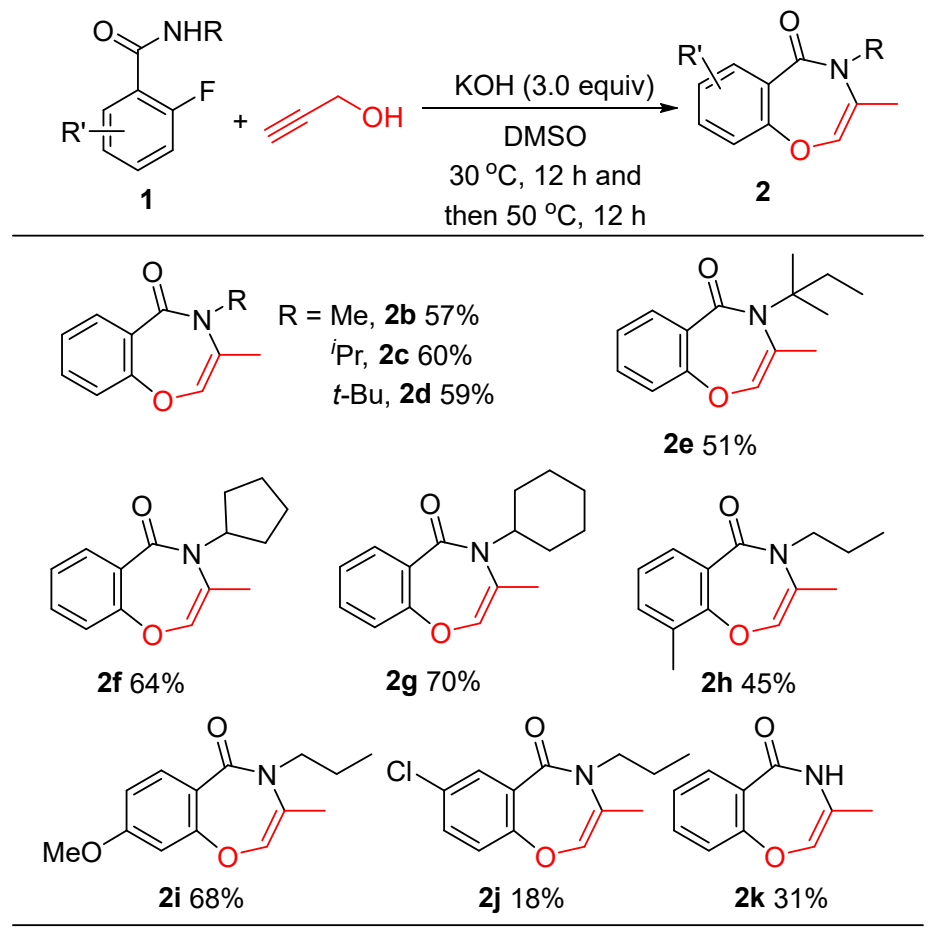

a The reactions were carried out using $0.5 \mathrm{mmol}$ of $\mathbf{1}, 0.6 \mathrm{mmol}$ of 2-propyn-1-ol, and $1.5 \mathrm{mmol}$ of $\mathrm{KOH}$ in anhydrous DMSO $(4.0 \mathrm{~mL})$.

Scheme 2. Formation of 1,4-benzoxazepin-5(4H)-ones in $\mathrm{KOH} / \mathrm{DMSO}^{a}$. 
We also examined the substrate scope under the reaction conditions indicated in entry 17 of Table 1 access to 1,3-benzoxazin-4(4H)-ones (3) by reacting 2-propyn-1-ol with different ortho-fluorobenzamides in $\mathrm{MeCN}$. As summarized in Scheme 3, the reactions of $\mathbf{1} \mathbf{b}-\mathbf{d}$ and $\mathbf{1} \mathbf{g}-\mathbf{j}$ afforded the corresponding 2-vinyl-1,3-benzoxazin-4(4H)-one derivatives $\mathbf{3 b}-\mathbf{d}$ and $\mathbf{3} \mathbf{g}-\mathbf{j}$ in good to high yields. It should be noted that, compared to $\mathrm{KOH} / \mathrm{DMSO}$ system, ortho-fluorobenzamides having electron-withdrawing group displayed a higher reactivity than ones with an electron-donating group (1j vs. $\mathbf{1 i}$ ) in $\mathrm{KOH} / \mathrm{MeCN}$ in undergoing the cyclization reaction to give the expected cyclic products in good yield ( $3 \mathbf{j}$ vs. $2 \mathbf{j}$ and $3 \mathbf{i}$ vs. 2i) (vide infra). In addition, both $\mathbf{1 1}$ and $\mathbf{1 m}$ also showed good reactivity, giving the corresponding products of $\mathbf{3 l}$ and $\mathbf{3 m}$ (vide supra). Note that in $\mathrm{KOH} / \mathrm{MeCN}$, all the reactions occurred with excellent chemoselectivity, and only a trace amount of the corresponding product $\mathbf{2}$ formed in the reaction mixtures.

However, when substituted propargyl alcohols, such as 1-methyl-2-propyn-1-ol, 1-phenyl2-propyn-1-ol, 3-methyl-2-propyn-1-ol, and 3-phenyl-2-propyn-1-ol, were subjected to the similar reaction conditions, although the formation of the corresponding cyclic compounds 2 and 3 could be determined by GC-MS, the reactions unfortunately occurred not only with low chemoselectivity in both DMSO and MeCN solvents, but also with low total yields of 2 and 3.

In order to understand the chemodivergent formation of 2 and $\mathbf{3}$ switched by solvents, the observation of real-time reactions of 1a with 2-propyn-1-ol in NMR tube using DMSO- $d_{6}$ or $\mathrm{CD}_{3} \mathrm{CN}$ were introduced. As shown in Scheme 4, in KOH/DMSO- $d_{6}$, the cyclization reaction occurred fast, and a $1 \mathrm{~h}$ reaction at $30{ }^{\circ} \mathrm{C}$ resulted in the formation of $\mathbf{2 a}$ and $3 \mathrm{a}$ in $58 \%$ and $10 \%$ NMR yields, respectively. In this case, no considerable amount of intermediates could be observed in ${ }^{1} \mathrm{H}-\mathrm{NMR}$. An additional 1 $\mathrm{h}$ reaction led to the yield increase of $\mathbf{2 a}$ to $74 \%$, whereas the NMR yield of $\mathbf{3 a}$ was decreased to $6 \%$. A subsequent $2 \mathrm{~h}$ reaction at $50{ }^{\circ} \mathrm{C}$ afforded 2a in $82 \%$ NMR yield, and 3a was determined in a small amount $(<5 \%)$. In addition, we also examined the conversion of $3 \mathbf{a}$ in $\mathrm{KOH} / \mathrm{DMSO}$ at $50{ }^{\circ} \mathrm{C}$ for $2 \mathrm{~h}$, and found that 3a completely disappeared due possibly to its polymerization, as the formation of $\mathbf{2 a}$ could not be observed at all in the reaction mixture. Therefore, it can be concluded that in the KOH/DMSO system, the excellent regioselectivity for the formation of $\mathbf{2 a}$ resulted from the easy formation of $\mathbf{2 a}$ and the side-reaction of $\mathbf{3 a}$.

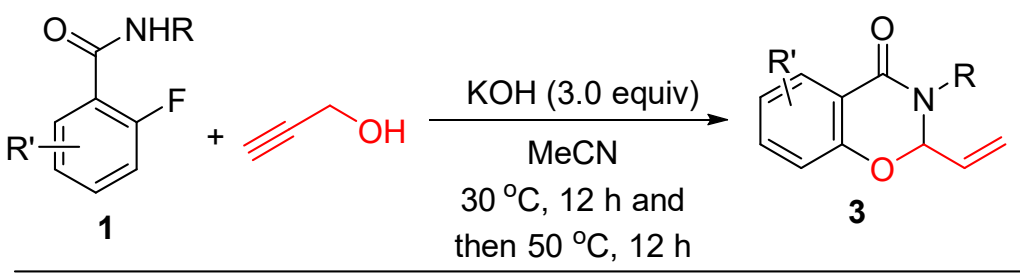

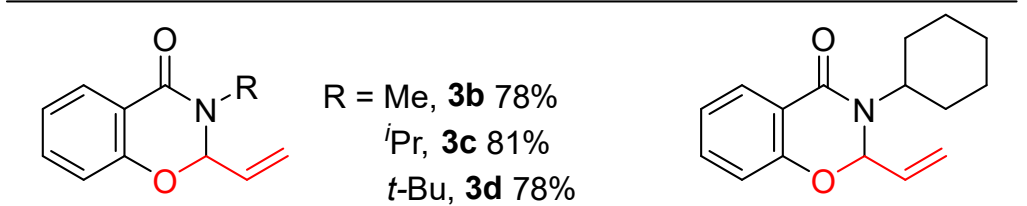

$3 \mathrm{~g} 85 \%$<smiles>[R]O[C@H]1Oc2ccc([R])cc2C(=O)N1CCC</smiles>

${ }^{a}$ The reactions were carried out using $0.5 \mathrm{mmol}$ of 1, $0.6 \mathrm{mmol}$ of 2-propyn-1-ol, and $1.5 \mathrm{mmol}$ of $\mathrm{KOH}$ in anhydrous $\mathrm{MeCN}(4.0 \mathrm{~mL})$.

Scheme 3. Formation of 1,3-benzoxazin-4(4H)-ones in $\mathrm{KOH} / \mathrm{MeCN}^{a}$. 
On the other hand, in $\mathrm{KOH} / \mathrm{CD}_{3} \mathrm{CN}$, the reaction of 1a with 2-propyn-1-ol in NMR tube without stirring occurred very slowly, and only small amount of intermediate 4 a could be determined from the $\mathrm{S}_{\mathrm{N}} \mathrm{Ar}$ reaction. 1a was almost remained, and neither $\mathbf{2 a}$ nor $3 \mathbf{a}$ formed at all at $30^{\circ} \mathrm{C}$ for $12 \mathrm{~h}$. Even a prolonged reaction time (at $30{ }^{\circ} \mathrm{C}$ for $36 \mathrm{~h}$ ) did not result in the formation of considerable amount of $3 \mathrm{a}$, and in this case, $4 \mathbf{a}$ was the major product. With the subsequent reaction at $50{ }^{\circ} \mathrm{C}$ for $12 \mathrm{~h}, 3 \mathbf{a}$ formed in $31 \%$ NMR yield, and in the sequent reaction for $36 \mathrm{~h}$, the yield of $3 \mathrm{a}$ was increased to $54 \%$.

Taking into consideration of the results shown in Schemes 2-4, it might be concluded that the chemodivergent formation of either 2 or $\mathbf{3}$ switched by using DMSO or MeCN as solvents resulted from the base strength of the reaction mixture. $\mathrm{KOH} / \mathrm{DMSO}$ was well-applied as the superbase medium to promote the diverse organic transformation due to the high solubility of $\mathrm{KOH}$ in DMSO [47], whereas $\mathrm{KOH} / \mathrm{MeCN}$ is a medium alkaline condition owing to the low solubility of $\mathrm{KOH}$ in $\mathrm{MeCN}$. In fact, the present reaction mixtures in DMSO were homogeneous to afford 2, but the reaction mixtures in $\mathrm{MeCN}$ were heterogeneous to give 3. Thus, it is also easy to understand why in NMR tube, without stirring, a very low rate of reaction in $\mathrm{CD}_{3} \mathrm{CN}$ was observed (Scheme 4 vs. Scheme 3).
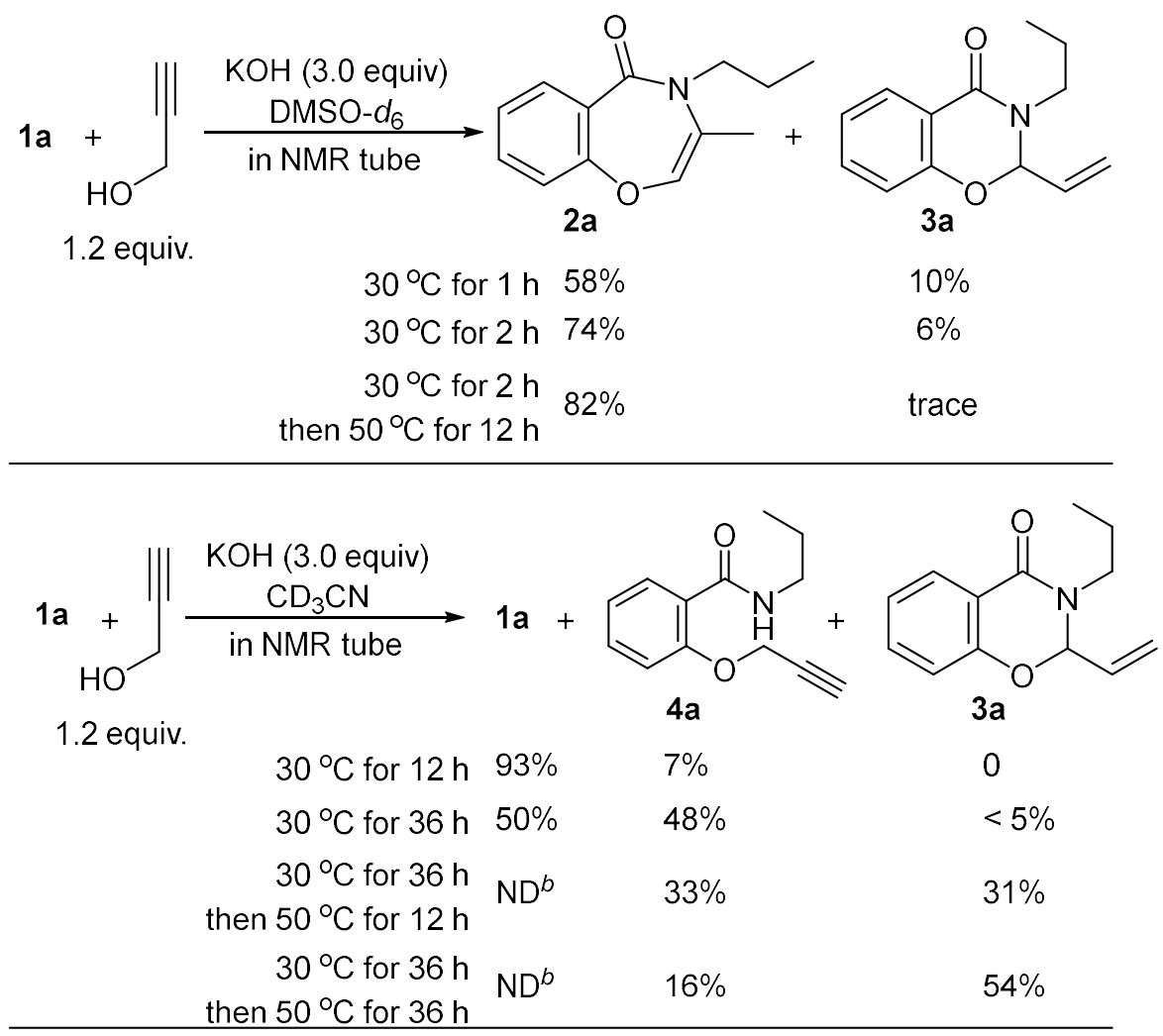

${ }^{a}$ NMR yield using 1,3,5-trimethoxybenzene as an internal standard. ${ }^{b} \mathrm{ND}$ : not determined due to the overlapping of peaks.

Scheme 4. Real-time monitoring of 1a with 2-propyn-1-ol reaction ${ }^{\text {a. }}$

On the basis of the obtained results and above discussion, a proposed mechanism for the formation of either $\mathbf{2}$ in DMSO or 3 in MeCN is depicted in Scheme 5. It involves the $\mathrm{S}_{\mathrm{N}} \mathrm{Ar}$ reaction of $\mathbf{1}$ with 2-propyn-1-ol, giving the intermediate of ortho-[(2-propynyl)oxy]benzamide 4 . In the KOH/DMSO system, a superbase medium [47], the formation of 4 and the subsequent formation of a nitrogen anion $\mathbf{5}$ are very fast and favorable, leading to the quickly intramolecular nucleophilic addition to alkyne concurrently to construct the seven-membered ring via a 7-exo-dig cyclization, and the final protonation step afforded 2. On the other hand, in the $\mathrm{KOH} / \mathrm{MeCN}$ system, a relatively weak base medium compared to $\mathrm{KOH} / \mathrm{DMSO}$, not only is the formation of 4 slow, but also the isomerization 
of 4 into allenyl intermediate 6 is possible [48], which undergoes the base-promoted intramolecular Michael addition of $\mathrm{N}-\mathrm{H}$ to allene giving 3 [49].

We also examined the conversion of the isolated intermediate $4 a$ under the similar reaction conditions as shown in Schemes 2 and 3. As expected, $2 \mathbf{a}$ and $3 \mathbf{a}$ could be isolated in $65 \%$ and $41 \%$ yields, respectively (Scheme 6).

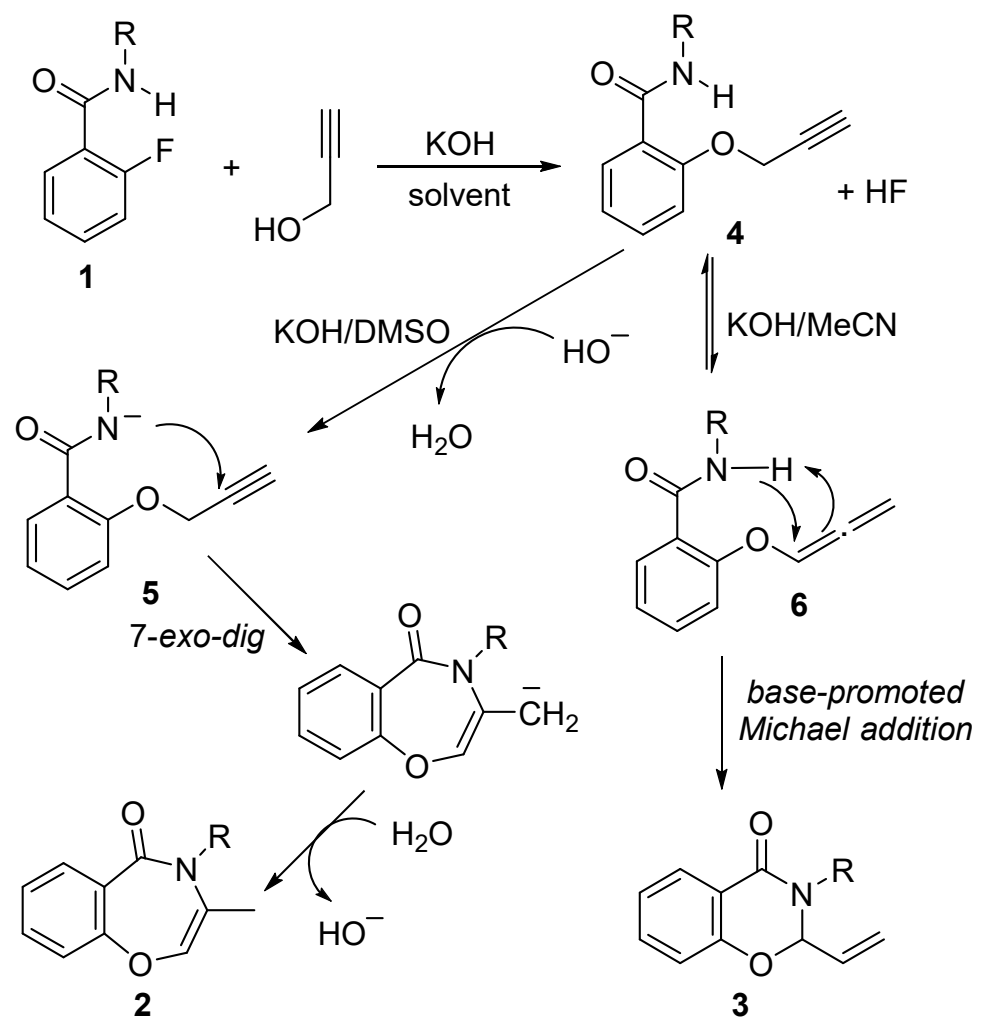

Scheme 5. Proposed mechanism for the chemodivergent formation of 1,4-benzoxazepin-5(4H)-ones and 1,3-benzoxazin-4(4H)-ones.

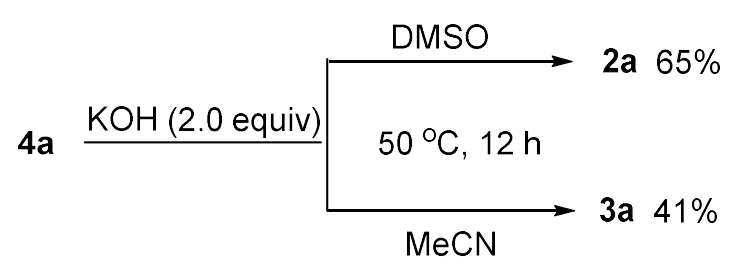

Scheme 6. Formation of $2 \mathbf{a}$ and $3 \mathbf{a}$ from intermediate $4 \mathbf{a}$.

\section{Materials and Methods}

\subsection{General Methods}

All commercial reagents and metal salts are analytically pure and used directly without further purification. $1 \mathbf{k}$ is commercially available and other ortho-fluoro- $N$-alkylbenzamides are known compounds and were prepared by a modified procedure via the reactions of ortho-fluorobenzoyl chlorides with primary amines (the procedure, yields in both weight and percentage, and ${ }^{1} \mathrm{H}-\mathrm{NMR}$ charts are reported in the Supplementary Information) [50]. KOH (99.99\%) was obtained from Sigma-Aldrich (St. Louis, MO, USA). Nuclear magnetic resonance (NMR) spectra were recorded on an ECA-400 spectrometer (JEOL, Tokyo, Japan) using $\mathrm{CDCl}_{3}$ as solvent at $298 \mathrm{~K} .{ }^{1} \mathrm{H}$ NMR $(400 \mathrm{MHz})$ chemical shifts $(\delta)$ were referenced to internal standard TMS (for $\left.{ }^{1} \mathrm{H}, \delta=0.00\right) .{ }^{13} \mathrm{C}$ NMR $(100 \mathrm{MHz})$ 
chemical shifts were referenced to internal solvent $\mathrm{CDCl}_{3}$ (for ${ }^{13} \mathrm{C}, \delta=77.16$ ). The high-resolution mass spectra (HRMS) with electron spray ionization (ESI) were obtained with a micrOTOF-Q spectrometer (Agilent, Santa Clara, CA, USA). The melting points were uncorrected. Single crystals of $\mathbf{2} \mathbf{g}$ and $\mathbf{3 g}$ were obtained by slow evaporation of their solution in a mixture solvent of acetone and $n$-hexane.

\subsection{Typical Experimental Procedure for the Synthesis of N-Propyl-3-methyl-1,4-benzoxazepin-5(4H)-one (2a)}

A mixture of 2-fluoro- $N$-propylbenzamide (1a, $90.5 \mathrm{mg}, 0.5 \mathrm{mmol}), 2$-propyl-1-ol (ca. $34.0 \mathrm{mg}$, $0.6 \mathrm{mmol}), \mathrm{KOH}(84.0 \mathrm{mg}, 1.5 \mathrm{mmol})$, and DMSO $(4.0 \mathrm{~mL})$ in a $25 \mathrm{~mL}$ screw-capped thick-walled Pyrex tube was stirred at $30^{\circ} \mathrm{C}$ for $12 \mathrm{~h}$, and then at $50{ }^{\circ} \mathrm{C}$ for $12 \mathrm{~h}$. After the reaction mixture was cooled to room temperature, water $(10 \mathrm{~mL})$ was added with stirring, and the mixture was extracted with ethyl acetate three times $(3 \times 10 \mathrm{~mL})$. The combined organic phases were dried over anhydrous $\mathrm{mgSO}_{4}$. The filtered solution was then concentrated under reduced pressure, and the crude residue was purified by column chromatography on silica gel with the use of petroleum ether/ethyl acetate (gradient mixture ratio from 20:1 to $4: 1$ in volume) to afford $2 \mathbf{a}$ as a pale yellow oil in $54 \%$ yield (58.5 $\mathrm{mg}$ ).

When $\mathrm{MeCN}$ was used as solvent to replace DMSO, the similar operation afforded $\mathrm{N}$-propyl-2vinyl-2,3-dihydro-1,3-benzoxazin-4(4H)-ones (3a) as a pale yellow oil in $83 \%$ yield (90.5 mg).

\subsection{Characterization Data of Products:}

N-Propyl-3-methyl-1,4-benzoxazepin-5(4H)-one (2a): Pale yellow oil (58.5 mg, 54\%). ${ }^{1} \mathrm{H}$ NMR (400 MHz, $\left.\mathrm{CDCl}_{3}\right): \delta 7.86(\mathrm{dd}, J=7.8,1.6 \mathrm{~Hz}, 1 \mathrm{H}), 7.40($ apparent td, $J=7.8,1.6 \mathrm{~Hz}, 1 \mathrm{H}), 7.18$ (apparent $\mathrm{t}, J=7.8 \mathrm{~Hz}$, $1 \mathrm{H}), 6.96(\mathrm{~d}, J=7.8 \mathrm{~Hz}, 1 \mathrm{H}), 5.43(\mathrm{~s}, 1 \mathrm{H}), 3.57(\mathrm{t}, J=7.4 \mathrm{~Hz}, 2 \mathrm{H}), 1.93(\mathrm{~s}, 3 \mathrm{H}), 1.64-1.73(\mathrm{~m}, 2 \mathrm{H}), 0.96(\mathrm{t}$, $J=7.4 \mathrm{~Hz}, 3 \mathrm{H}) .{ }^{13} \mathrm{C}$ NMR $\left(100 \mathrm{MHz}, \mathrm{CDCl}_{3}\right): \delta 166.6,160.5,148.5,133.1,132.2,127.3,124.8,120.1,115.0$, 49.8, 21.4, 17.7, 11.2. HRMS (ESI) $m / z$ : $[\mathrm{M}+\mathrm{H}]^{+}$calcd for $\mathrm{C}_{13} \mathrm{H}_{16} \mathrm{NO}_{2}, 218.1176$; found, 218.1174 .

N-Methyl-3-methyl-1,4-benzoxazepin-5(4H)-one (2b): Pale yellow oil (54.1 mg, 57\%). ${ }^{1} \mathrm{H}$ NMR $(400 \mathrm{MHz}$, $\left.\mathrm{CDCl}_{3}\right) \delta 7.87(\mathrm{dd}, J=7.8,1.8 \mathrm{~Hz}, 1 \mathrm{H}), 7.41$ (apparent td, $\left.J=7.8,1.8 \mathrm{~Hz}, 1 \mathrm{H}\right), 7.19$ (apparent td, $J=7.8$, $1.8 \mathrm{~Hz}, 1 \mathrm{H}), 6.97(\mathrm{dd}, J=7.8,1.8 \mathrm{~Hz}, 1 \mathrm{H}), 5.46(\mathrm{~s}, 1 \mathrm{H}), 3.19(\mathrm{~s}, 3 \mathrm{H}), 1.93(\mathrm{~s}, 3 \mathrm{H}) .{ }^{13} \mathrm{C}$ NMR $(100 \mathrm{MHz}$, $\left.\mathrm{CDCl}_{3}\right) \delta 166.8,160.4,147.9,133.1,132.1,126.9,124.8,120.1,115.8,35.9,17.5$. HRMS (ESI) $m / z:[\mathrm{M}+\mathrm{H}]^{+}$ calcd for $\mathrm{C}_{11} \mathrm{H}_{12} \mathrm{NO}_{2}, 190.0863$; found, 190.0862 .

N-Isopropyl-3-methyl-1,4-benzoxazepin-5(4H)-one (2c): Pale yellow oil (64.7 mg, 60\%). ${ }^{1} \mathrm{H}$ NMR $(400 \mathrm{MHz}$, $\left.\mathrm{CDCl}_{3}\right): \delta 7.88(\mathrm{dd}, J=7.8,1.6 \mathrm{~Hz}, 1 \mathrm{H}), 7.39$ (apparent td, $\left.J=7.8,1.6 \mathrm{~Hz}, 1 \mathrm{H}\right), 7.18$ (apparent td, $J=7.8$, $1.6 \mathrm{~Hz}, 1 \mathrm{H}), 6.96(\mathrm{dd}, J=7.8,1.6 \mathrm{~Hz}, 1 \mathrm{H}), 5.49(\mathrm{~s}, 1 \mathrm{H}), 5.04$ (hept, $J=6.8 \mathrm{~Hz}, 1 \mathrm{H}), 1.95(\mathrm{~s}, 3 \mathrm{H}), 1.23(\mathrm{~d}$, $J=6.8 \mathrm{~Hz}, 6 \mathrm{H}) .{ }^{13} \mathrm{C} \mathrm{NMR}\left(100 \mathrm{MHz}, \mathrm{CDCl}_{3}\right): \delta 166.2,160.5,149.8,132.9,132.4,127.3,124.7,112.0,109.9$, 45.7, 20.3, 17.7. HRMS (ESI) $\mathrm{m} / z$ : $[\mathrm{M}+\mathrm{H}]^{+}$calcd for $\mathrm{C}_{13} \mathrm{H}_{16} \mathrm{NO}_{2}, 218.1176$; found, 218.1173 .

N-t-Butyl-3-methyl-1,4-benzoxazepin-5(4H)-one (2d): Pale yellow oil (68.2 mg, 59\%). ${ }^{1} \mathrm{H}$ NMR $(400 \mathrm{MHz}$, $\left.\mathrm{CDCl}_{3}\right): \delta 7.92(\mathrm{dd}, J=7.8,1.6 \mathrm{~Hz}, 1 \mathrm{H}), 7.37$ (apparent td, $\left.J=7.8,1.6 \mathrm{~Hz}, 1 \mathrm{H}\right), 7.17$ (apparent td, $J=7.8$, $1.6 \mathrm{~Hz}, 1 \mathrm{H}), 6.96(\mathrm{dd}, J=7.8,1.6 \mathrm{~Hz}, 1 \mathrm{H}), 5.59(\mathrm{q}, J=0.8 \mathrm{~Hz}, 1 \mathrm{H}), 1.90(\mathrm{~d}, J=0.8 \mathrm{~Hz}, 3 \mathrm{H}), 1.54(\mathrm{~s}$, 9H). ${ }^{13} \mathrm{C} \mathrm{NMR}\left(100 \mathrm{MHz}, \mathrm{CDCl}_{3}\right): \delta 166.7,161.2,150.0,132.5,128.2,124.5,119.7,113.7,58.9,28.7,17.2$. HRMS (ESI) $m / z$ : $[\mathrm{M}+\mathrm{H}]^{+}$calcd for $\mathrm{C}_{14} \mathrm{H}_{18} \mathrm{NO}_{2}, 232.1332$; found, 232.1332 .

N-t-Amyl-3-methyl-1,4-benzoxazepin-5(4H)-one (2e): Pale yellow oil (62.1 mg, 51\%). ${ }^{1} \mathrm{H}$ NMR (400 MHz, $\left.\mathrm{CDCl}_{3}\right): \delta 7.90(\mathrm{dd}, J=7.8,1.6 \mathrm{~Hz}, 1 \mathrm{H}), 7.36$ (apparent td, $\left.J=7.8,1.6 \mathrm{~Hz}, 1 \mathrm{H}\right), 7.16$ (apparent td, $J=7.8$, $1.6 \mathrm{~Hz}, 1 \mathrm{H}), 6.96(\mathrm{dd}, J=7.8,1.6 \mathrm{~Hz}, 1 \mathrm{H}), 5.56(\mathrm{~s}, 1 \mathrm{H}), 2.02(\mathrm{q}, J=7.5 \mathrm{~Hz}, 2 \mathrm{H}), 1.89(\mathrm{~s}, 3 \mathrm{H}), 1.48(\mathrm{~s}, 6 \mathrm{H})$, $0.89(\mathrm{t}, J=7.5 \mathrm{~Hz}, 3 \mathrm{H}) .{ }^{13} \mathrm{C} \mathrm{NMR}\left(100 \mathrm{MHz}, \mathrm{CDCl}_{3}\right): \delta 166.8,161.3,150.2,132.5,128.3,124.6,119.7,114.3$, 62.0, 32.4, 27.0, 17.2, 8.6. HRMS (ESI) $m / z$ : $[\mathrm{M}+\mathrm{H}]^{+}$calcd for $\mathrm{C}_{15} \mathrm{H}_{20} \mathrm{NO}_{2}, 246.1489$; found, 246.1487 .

N-Cyclopentyl-3-methyl-1,4-benzoxazepin-5(4H)-one (2f): Pale yellow oil (78.1 mg, 64\%). ${ }^{1} \mathrm{H}$ NMR $\left(400 \mathrm{MHz}, \mathrm{CDCl}_{3}\right): \delta 7.86(\mathrm{dd}, J=7.8,1.6 \mathrm{~Hz}, 1 \mathrm{H}), 7.37$ (apparent td, $\left.J=7.8,1.6 \mathrm{~Hz}, 1 \mathrm{H}\right), 7.16$ (apparent $\mathrm{td}, J=7.8,1.6 \mathrm{~Hz}, 1 \mathrm{H}), 6.94(\mathrm{dd}, J=7.8,1.6 \mathrm{~Hz}, 1 \mathrm{H}), 5.44(\mathrm{~s}, 1 \mathrm{H}), 5.10$ (pent, $J=8.2 \mathrm{~Hz}, 1 \mathrm{H}), 2.00-1.95$ (m, 2H), $1.93(\mathrm{~s}, 3 \mathrm{H}), 1.74-1.53(\mathrm{~m}, 6 \mathrm{H}) .{ }^{13} \mathrm{C} \mathrm{NMR}\left(100 \mathrm{MHz}, \mathrm{CDCl}_{3}\right): \delta 166.7,160.5,149.8,132.9,132.5$, 
127.3, 124.7, 112.0, 110.8, 55.6, 29.6, 24.6, 17.8. HRMS (ESI) $m / z:[\mathrm{M}+\mathrm{H}]^{+}$calcd for $\mathrm{C}_{15} \mathrm{H}_{18} \mathrm{NO}_{2}, 244.1332$; found, 244.1331 .

N-Cyclohexyl-3-methyl-1,4-benzoxazepin-5(4H)-one (2g): Pale yellow solid (89.4 mg, 70\%). mp 63-65 ${ }^{\circ} \mathrm{C}$. ${ }^{1} \mathrm{H}$ NMR $\left(400 \mathrm{MHz}, \mathrm{CDCl}_{3}\right): \delta 7.87(\mathrm{dd}, J=7.8,1.6 \mathrm{~Hz}, 1 \mathrm{H}), 7.40$ (apparent td, $\left.J=7.8,1.6 \mathrm{~Hz}, 1 \mathrm{H}\right), 7.19$ (apparent td, $J=7.8,1.6 \mathrm{~Hz}, 1 \mathrm{H}), 6.96(\mathrm{dd}, J=7.8,1.6 \mathrm{~Hz}, 1 \mathrm{H}), 5.51(\mathrm{q}, J=0.9 \mathrm{~Hz}, 1 \mathrm{H}), 4.67-4.55(\mathrm{~m}, 1 \mathrm{H})$, $1.94(\mathrm{~d}, J=0.9 \mathrm{~Hz}, 3 \mathrm{H}), 1.91-1.78(\mathrm{~m}, 4 \mathrm{H}), 1.73-1.62(\mathrm{~m}, 2 \mathrm{H}), 1.50-1.40(\mathrm{~m}, 4 \mathrm{H}) .{ }^{13} \mathrm{C} \mathrm{NMR}(100 \mathrm{MHz}$, $\left.\mathrm{CDCl}_{3}\right): \delta 166.2,160.5,149.3,132.8,132.4,127.4,124.7,119.9,110.9,53.9,30.7,25.8,25.6,17.7$. HRMS (ESI) $m / z:[\mathrm{M}+\mathrm{H}]^{+}$calcd for $\mathrm{C}_{16} \mathrm{H}_{20} \mathrm{NO}_{2}$, 258.1489; found, 258.1488 .

N-Propyl-3,9-dimethyl-1,4-benzoxazepin-5(4H)-one (2h): Pale yellow oil (52.3 $\mathrm{mg}, 45 \%) .{ }^{1} \mathrm{H}$ NMR $\left(400 \mathrm{MHz}, \mathrm{CDCl}_{3}\right): \delta 7.67(\mathrm{dd}, J=7.8,1.5 \mathrm{~Hz}, 1 \mathrm{H}), 7.27(\mathrm{~d}, J=7.8 \mathrm{~Hz}, 1 \mathrm{H}), 7.07$ (apparent t $J=7.8 \mathrm{~Hz}$, $1 \mathrm{H}), 5.45(\mathrm{~s}, 1 \mathrm{H}), 3.57(\mathrm{t}, J=7.4 \mathrm{~Hz}, 2 \mathrm{H}), 2.32(\mathrm{~s}, 3 \mathrm{H}), 1.97(\mathrm{~s}, 3 \mathrm{H}), 1.76-1.65(\mathrm{~m}, 2 \mathrm{H}), 0.96(\mathrm{t}, J=7.4 \mathrm{~Hz}$, 3H). ${ }^{13} \mathrm{C}$ NMR $\left(100 \mathrm{MHz}, \mathrm{CDCl}_{3}\right): \delta 167.0,159.3,148.7,134.3,129.9,129.0,127.4,124.3,115.5,49.9,21.5$, 18.3, 16.1, 11.3. HRMS (ESI) $m / z$ : [M + H] ${ }^{+}$calcd for $\mathrm{C}_{14} \mathrm{H}_{18} \mathrm{NO}_{2}, 232.1332$; found, 232.1331.

8-Methoxyl-N-propyl-3-methyl-1,4-benzoxazepin-5(4H)-one (2i): Pale yellow oil (84.1 mg, 68\%). ${ }^{1} \mathrm{H}$ NMR $\left(400 \mathrm{MHz}, \mathrm{CDCl}_{3}\right): \delta 7.81(\mathrm{~d}, J=8.7 \mathrm{~Hz}, 1 \mathrm{H}), 6.73(\mathrm{dd}, J=8.7,2.8 \mathrm{~Hz} 1 \mathrm{H}), 6.48(\mathrm{~d}, J=2.8 \mathrm{~Hz}, 1 \mathrm{H}), 5.43$ $(\mathrm{s}, 1 \mathrm{H}), 3.83(\mathrm{~s}, 3 \mathrm{H}), 3.55(\mathrm{t}, J=7.4 \mathrm{~Hz}, 2 \mathrm{H}), 1.94(\mathrm{~s}, 3 \mathrm{H}), 1.75-1.62(\mathrm{~m}, 2 \mathrm{H}), 0.96(\mathrm{td}, J=7.4,3.5 \mathrm{~Hz}, 3 \mathrm{H})$. ${ }^{13} \mathrm{C}$ NMR (100 MHz, $\left.\mathrm{CDCl}_{3}\right): \delta 166.2,163.7,161.6,147.7,133.5,119.5,115.1,111.0,105.1,55.7,49.8,21.5$, 17.8, 11.3. HRMS (ESI) $m / z:[\mathrm{M}+\mathrm{H}]^{+}$calcd for $\mathrm{C}_{14} \mathrm{H}_{18} \mathrm{NO}_{3}$, 248.1281; found, 248.1279.

7-Chloro-N-propyl-3-methyl-1,4-benzoxazepin-5(4H)-one (2j): Pale yellow oil (22.3 mg, 18\%). ${ }^{1} \mathrm{H}$ NMR $\left(400 \mathrm{MHz}, \mathrm{CDCl}_{3}\right): \delta 7.83(\mathrm{~d}, J=2.8 \mathrm{~Hz}, 1 \mathrm{H}), 7.34(\mathrm{dd}, J=8.0,2.8 \mathrm{~Hz}, 1 \mathrm{H}), 6.91(\mathrm{~d}, J=8.0 \mathrm{~Hz}, 1 \mathrm{H})$, $5.43(\mathrm{~s}, 1 \mathrm{H}), 3.56(\mathrm{t}, J=7.4 \mathrm{~Hz}, 2 \mathrm{H}), 1.93(\mathrm{~s}, 3 \mathrm{H}), 1.70-1.62(\mathrm{~m}, 2 \mathrm{H}), 0.96(\mathrm{t}, J=7.4 \mathrm{~Hz}, 3 \mathrm{H}) .{ }^{13} \mathrm{C} \mathrm{NMR}$ $\left(100 \mathrm{MHz}, \mathrm{CDCl}_{3}\right): \delta 165.3,159.0,148.8,132.9,131.9,130.2,128.6,121.6,115.0,50.0,21.4,17.7,11.3$. HRMS (ESI) $m / z:[\mathrm{M}+\mathrm{H}]^{+}$calcd for $\mathrm{C}_{13} \mathrm{H}_{15} \mathrm{ClNO}_{2}, 252.0786$; found, 252.0786 .

3-Methyl-1,4-benzoxazepin-5(4H)-one (2k) [23]: Pale yellow oil (26.9 mg, 31\%). ${ }^{1} \mathrm{H}$ NMR (400 MHz, $\left.\mathrm{CDCl}_{3}\right): \delta 11.18(\mathrm{~s}, 1 \mathrm{H}), 7.71(\mathrm{dd}, J=7.8,1.6 \mathrm{~Hz}, 1 \mathrm{H}), 7.24(\mathrm{ddd}, J=8.3,7.8,1.6 \mathrm{~Hz}, 1 \mathrm{H}), 6.97(\mathrm{dd}, J=7.8$, $1.6 \mathrm{~Hz}, 1 \mathrm{H}), 6.88(\mathrm{ddd}, J=8.3,7.8,1.6 \mathrm{~Hz}, 1 \mathrm{H}), 6.75(\mathrm{q}, J=1.2 \mathrm{~Hz}, 1 \mathrm{H}), 2.33(\mathrm{~d}, J=1.2 \mathrm{~Hz}, 3 \mathrm{H}) .{ }^{13} \mathrm{C}$ NMR (100 MHz, $\left.\mathrm{CDCl}_{3}\right): \delta 160.6,157.1,147.9,131.9,125.7,122.1,119.4,117.2,111.5,11.0$.

N-Propyl-2-vinyl-2,3-dihydro-1,3-benzoxazin-4(4H)-one (3a): Pale yellow oil (90.5 mg, 83\%). ${ }^{1} \mathrm{H}$ NMR $\left(400 \mathrm{MHz}, \mathrm{CDCl}_{3}\right): \delta 7.93(\mathrm{dd}, J=7.8,1.8 \mathrm{~Hz}, 1 \mathrm{H}), 7.41$ (apparent td, $J=7.8,1.6 \mathrm{~Hz}, 1 \mathrm{H}$ ), 7.07 (apparent $\mathrm{td}, J=7.8,1.6 \mathrm{~Hz}, 1 \mathrm{H}), 6.91(\mathrm{dd}, J=7.8,1.8 \mathrm{~Hz}, 1 \mathrm{H}), 5.97(\mathrm{ddd}, J=16.8,10.2,5.8 \mathrm{~Hz}, 1 \mathrm{H}), 5.64(\mathrm{~d}$, $J=5.8 \mathrm{~Hz}, 1 \mathrm{H}), 5.40(\mathrm{~d}, J=16.8 \mathrm{~Hz}, 1 \mathrm{H}), 5.36(\mathrm{~d}, J=10.2 \mathrm{~Hz}, 1 \mathrm{H}), 3.93(\mathrm{ddd}, J=14.0,8.0,6.8 \mathrm{~Hz}, 1 \mathrm{H})$, $2.97(\mathrm{ddd}, J=14.0,8.0,6.8 \mathrm{~Hz}, 1 \mathrm{H}), 1.77-1.62(\mathrm{~m}, 2 \mathrm{H}), 0.97(\mathrm{t}, J=7.4 \mathrm{~Hz}, 3 \mathrm{H}) .{ }^{13} \mathrm{C} \mathrm{NMR}(100 \mathrm{MHz}$, $\left.\mathrm{CDCl}_{3}\right): \delta 161.4,155.4,134.1,132.6,128.0,122.4,120.9,118.7,116.8,87.7,45.8,21.7,11.4$. HRMS (ESI) $m / z:[\mathrm{M}+\mathrm{H}]^{+}$calcd for $\mathrm{C}_{13} \mathrm{H}_{16} \mathrm{NO}_{2}, 218.1176$; found, 218.1175.

N-Methyl-2-vinyl-2,3-dihydro-1,3-benzoxazin-4(4H)-one (3b) [45]: Pale yellow oil (73.7 $\mathrm{mg}, 78 \%) .{ }^{1} \mathrm{H}$ NMR $\left(400 \mathrm{MHz}, \mathrm{CDCl}_{3}\right): \delta 7.93(\mathrm{dd}, J=7.8,1.7 \mathrm{~Hz}, 1 \mathrm{H}), 7.41$ (apparent td, $\left.J=7.8,1.7 \mathrm{~Hz}, 1 \mathrm{H}\right), 7.07$ (apparent td, $J=7.8,1.7 \mathrm{~Hz}, 1 \mathrm{H}), 6.92(\mathrm{dd}, J=7.8,1.7 \mathrm{~Hz}, 1 \mathrm{H}), 5.97(\mathrm{ddd}, J=16.8,10.2,6.0 \mathrm{~Hz}, 1 \mathrm{H})$, $5.61(\mathrm{~d}, J=6.0 \mathrm{~Hz}, 1 \mathrm{H}), 5.45-5.34(\mathrm{~m}, 2 \mathrm{H}), 3.07(\mathrm{~s}, 3 \mathrm{H}) .{ }^{13} \mathrm{C} \mathrm{NMR}\left(100 \mathrm{MHz}, \mathrm{CDCl}_{3}\right): \delta 161.8,155.5$, 134.2, 131.9, 128.0, 122.4, 121.1, 118.3, 116.8, 89.2, 31.1. HRMS (ESI) $m / z:[\mathrm{M}+\mathrm{H}]^{+}$calcd for $\mathrm{C}_{11} \mathrm{H}_{12} \mathrm{NO}_{2}$, 190.0863; found, 190.0861 .

N-Isopropyl-2-vinyl-2,3-dihydro-1,3-benzoxazin-4(4H)-one (3c): Pale yellow oil (89.0 mg, 81\%). ${ }^{1} \mathrm{H}$ NMR $\left(400 \mathrm{MHz}, \mathrm{CDCl}_{3}\right): \delta 7.92(\mathrm{dd}, J=7.8,1.6 \mathrm{~Hz}, 1 \mathrm{H}), 7.40$ (apparent td, $\left.J=7.8,1.6 \mathrm{~Hz}, 1 \mathrm{H}\right), 7.05$ (apparent $\mathrm{td}, J=7.8,1.6 \mathrm{~Hz}, 1 \mathrm{H}), 6.88(\mathrm{dd}, J=7.8,1.6 \mathrm{~Hz}, 1 \mathrm{H}), 5.97(\mathrm{ddd}, J=16.8,10.0,6.0 \mathrm{~Hz}, 1 \mathrm{H}), 5.78(\mathrm{~d}$, $J=6.0 \mathrm{~Hz}, 1 \mathrm{H}), 5.40(\mathrm{~d}, J=16.8 \mathrm{~Hz}, 1 \mathrm{H}), 5.29(\mathrm{~d}, J=10.0 \mathrm{~Hz}, 1 \mathrm{H}), 4.85$ (hept, $J=6.8 \mathrm{~Hz}, 1 \mathrm{H}), 1.33$ $(\mathrm{d}, J=6.8 \mathrm{~Hz}, 3 \mathrm{H}), 1.21(\mathrm{~d}, J=6.8 \mathrm{~Hz}, 3 \mathrm{H}) .{ }^{13} \mathrm{C} \mathrm{NMR}\left(100 \mathrm{MHz}, \mathrm{CDCl}_{3}\right): \delta 160.7,154.9,134.6,133.9$, 128.0, 122.2, 120.5, 119.5, 116.7, 82.9, 45.0, 20.9, 20.7. HRMS (ESI) $m / z:[\mathrm{M}+\mathrm{H}]^{+}$calcd for $\mathrm{C}_{13} \mathrm{H}_{16} \mathrm{NO}_{2}$, 218.1176; found, 218.1175 . 
N-t-Butyl-2-vinyl-2,3-dihydro-1,3-benzoxazin-4(4H)-one (3d): Pale yellow oil (89.9 mg, 78\%). ${ }^{1} \mathrm{H}$ NMR $\left(400 \mathrm{MHz}, \mathrm{CDCl}_{3}\right): \delta 7.90(\mathrm{dd}, J=7.8,1.6 \mathrm{~Hz}, 1 \mathrm{H}), 7.38$ (apparent td, $\left.J=7.8,1.6 \mathrm{~Hz}, 1 \mathrm{H}\right), 7.03$ (apparent $\mathrm{td}, J=7.8,1.6 \mathrm{~Hz}, 1 \mathrm{H}), 6.86(\mathrm{dd}, J=7.8,1.6 \mathrm{~Hz}, 1 \mathrm{H}), 6.05-5.92(\mathrm{~m}, 2 \mathrm{H}), 5.41(\mathrm{~d}, J=16.8 \mathrm{~Hz}, 1 \mathrm{H}), 5.29(\mathrm{~d}$, $J=10.0 \mathrm{~Hz}, 1 \mathrm{H}), 1.56(\mathrm{~s}, 9 \mathrm{H}) .{ }^{13} \mathrm{C} \mathrm{NMR}\left(100 \mathrm{MHz}, \mathrm{CDCl}_{3}\right): \delta 162.1,154.7,134.9,133.8,127.9,122.1,120.5$, 120.4, 116.4, 84.6, 57.4, 28.8. HRMS (ESI) $m / z$ : $[\mathrm{M}+\mathrm{H}]^{+}$calcd for $\mathrm{C}_{14} \mathrm{H}_{18} \mathrm{NO}_{2}, 232.1332$; found, 232.1331.

N-Cyclohexyl-2-vinyl-2,3-dihydro-1,3-benzoxazin-4(4H)-one (3g): White solid (109.6 mg, 85\%). mp 84-86 ${ }^{\circ} \mathrm{C} .{ }^{1} \mathrm{H}$ NMR $\left(400 \mathrm{MHz}, \mathrm{CDCl}_{3}\right): \delta 7.91(\mathrm{~d}, J=7.8 \mathrm{~Hz}, 1 \mathrm{H}), 7.39$ (apparent $\mathrm{t}, J=7.8 \mathrm{~Hz}, 1 \mathrm{H}$ ), 7.05 (apparent $\mathrm{t}, J=7.8 \mathrm{~Hz}, 1 \mathrm{H}), 6.88(\mathrm{~d}, J=7.8 \mathrm{~Hz}, 1 \mathrm{H}), 5.95(\mathrm{ddd}, J=16.8,10.6,5.7 \mathrm{~Hz}, 1 \mathrm{H}), 5.80$ $(\mathrm{d}, J=5.7 \mathrm{~Hz}, 1 \mathrm{H}), 5.40(\mathrm{~d}, J=16.8 \mathrm{~Hz}, 1 \mathrm{H}), 5.28(\mathrm{~d}, J=10.6 \mathrm{~Hz}, 1 \mathrm{H}), 4.48(\mathrm{tt}, J=12.2,3.4 \mathrm{~Hz}, 1 \mathrm{H})$, 1.98-1.01 (m, 10H). ${ }^{13} \mathrm{C}$ NMR (100 MHz, $\left.\mathrm{CDCl}_{3}\right): \delta 160.8,155.0,134.7,134.0,128.1,122.3,120.1,119.7$, $116.8,83.2,52.9,31.4,31.2,26.0,25.9,25.6$. HRMS (ESI) $m / z$ : $[\mathrm{M}+\mathrm{H}]^{+}$calcd for $\mathrm{C}_{16} \mathrm{H}_{20} \mathrm{NO}_{2}, 258.1489$; found, 258.1487 .

N-Propyl-2-vinyl-8-methyl-2,3-dihydro-1,3-benzoxazin-4(4H)-one (3h): Pale yellow oil (65.8 mg, $54 \%) .{ }^{1} \mathrm{H}$ NMR $\left(400 \mathrm{MHz}, \mathrm{CDCl}_{3}\right): \delta 7.77(\mathrm{dd}, J=7.8,1.5 \mathrm{~Hz}, 1 \mathrm{H}), 7.25(\mathrm{dd}, J=7.8,1.5 \mathrm{~Hz}, 1 \mathrm{H}), 6.95$ (apparent t, $J=7.8 \mathrm{~Hz}, 1 \mathrm{H}), 5.95(\mathrm{ddd}, J=17.0,10.6,6.4 \mathrm{~Hz}, 1 \mathrm{H}), 5.67(\mathrm{~d}, J=6.4 \mathrm{~Hz}, 1 \mathrm{H}), 5.38(\mathrm{~d}, J=17.0 \mathrm{~Hz}, 1 \mathrm{H})$, $5.32(\mathrm{~d}, J=10.6 \mathrm{~Hz}, 1 \mathrm{H}), 3.94(\mathrm{ddd}, J=14.0,8.0,6.4 \mathrm{~Hz}, 1 \mathrm{H}), 2.97(\mathrm{ddd}, J=14.0,8.0,6.4 \mathrm{~Hz}, 1 \mathrm{H}), 2.21$ $(\mathrm{s}, 3 \mathrm{H}), 1.78-1.59(\mathrm{~m}, 2 \mathrm{H}), 0.97(\mathrm{t}, J=7.4 \mathrm{~Hz}, 3 \mathrm{H}) .{ }^{13} \mathrm{C} \mathrm{NMR}\left(100 \mathrm{MHz}, \mathrm{CDCl}_{3}\right): \delta 161.6,153.5,135.0$, $132.7,126.0,125.4,121.8,120.4,118.3,87.4,45.7,21.6,15.2,11.4$. HRMS (ESI) $m / z:[\mathrm{M}+\mathrm{H}]^{+}$calcd for $\mathrm{C}_{14} \mathrm{H}_{18} \mathrm{NO}_{2}$, 232.1332; found, 232.1331.

7-Methoxyl-N-propyl-2-vinyl-2,3-dihydro-1,3-benzoxazin-4(4H)-one (3i): Pale yellow oil (55.5 mg, 45\%). ${ }^{1} \mathrm{H}$ NMR $\left(400 \mathrm{MHz}, \mathrm{CDCl}_{3}\right): \delta 7.84(\mathrm{~d}, J=8.8 \mathrm{~Hz}, 1 \mathrm{H}), 6.62(\mathrm{dd}, J=8.8,2.4 \mathrm{~Hz}, 1 \mathrm{H}), 6.40(\mathrm{~d}, J=2.4 \mathrm{~Hz}$, $1 \mathrm{H}), 5.98(\mathrm{ddd}, J=16.8,10.4,5.9 \mathrm{~Hz}, 1 \mathrm{H}), 5.61(\mathrm{~d}, J=5.9 \mathrm{~Hz}, 1 \mathrm{H}), 5.40(\mathrm{~d}, J=16.8 \mathrm{~Hz}, 1 \mathrm{H}), 5.35(\mathrm{~d}$, $J=10.4 \mathrm{~Hz}, 1 \mathrm{H}), 3.91(\mathrm{ddd}, J=14.0,8.0,6.8 \mathrm{~Hz}, 1 \mathrm{H}), 3.81(\mathrm{~s}, 3 \mathrm{H}), 2.95(\mathrm{ddd}, J=14.0,8.0,6.8 \mathrm{~Hz}, 1 \mathrm{H})$, $1.72-1.58(\mathrm{~m}, 2 \mathrm{H}), 0.96(\mathrm{t}, J=7.4 \mathrm{~Hz}, 3 \mathrm{H}) .{ }^{13} \mathrm{C} \mathrm{NMR}\left(100 \mathrm{MHz}, \mathrm{CDCl}_{3}\right): \delta 164.5,161.5,157.1,132.6,129.5$, $120.8,111.8,109.4,101.1,88.0,55.7,45.6,21.7,11.4$. HRMS (ESI) $m / z:[\mathrm{M}+\mathrm{H}]^{+}$calcd for $\mathrm{C}_{14} \mathrm{H}_{18} \mathrm{NO}_{3}$, 248.1281; found, 248.1279 .

6-Chloro-N-propyl-2-vinyl-2,3-dihydro-1,3-benzoxazin-4(4H)-one (3j): Pale yellow oil (76.2 $\mathrm{mg}, 61 \%) .{ }^{1} \mathrm{H}$ $\operatorname{NMR}\left(400 \mathrm{MHz}, \mathrm{CDCl}_{3}\right): \delta 7.89(\mathrm{~d}, J=2.7 \mathrm{~Hz}, 1 \mathrm{H}), 7.35(\mathrm{dd}, J=8.7,2.7 \mathrm{~Hz}, 1 \mathrm{H}), 6.86(\mathrm{~d}, J=8.7 \mathrm{~Hz}, 1 \mathrm{H})$, $5.94(\mathrm{ddd}, J=17.0,10.3,5.5 \mathrm{~Hz}, 1 \mathrm{H}), 5.65(\mathrm{~d}, J=5.5 \mathrm{~Hz}, 1 \mathrm{H}), 5.40(\mathrm{~d}, J=17.0 \mathrm{~Hz}, 1 \mathrm{H}), 5.38(\mathrm{~d}, J=10.3 \mathrm{~Hz}$, $1 \mathrm{H}), 3.92(\mathrm{ddd}, J=14.0,8.4,7.2 \mathrm{~Hz}, 1 \mathrm{H}), 2.96(\mathrm{ddd}, J=14.0,8.4,7.2 \mathrm{~Hz}, 1 \mathrm{H}), 1.72-1.63(\mathrm{~m}, 2 \mathrm{H}), 0.97(\mathrm{t}$, $J=7.4 \mathrm{~Hz}, 3 \mathrm{H}) .{ }^{13} \mathrm{C} \mathrm{NMR}\left(100 \mathrm{MHz}, \mathrm{CDCl}_{3}\right): \delta 160.3,153.9,133.9,132.2,127.8,127.7,121.3,119.9,118.4$, 87.7, 46.0, 21.6, 11.4. HRMS (ESI) $m / z:[\mathrm{M}+\mathrm{H}]^{+}$calcd for $\mathrm{C}_{13} \mathrm{H}_{15} \mathrm{ClNO}_{2}, 252.0786$; found, 252.0785 .

6-Nitro-N-propyl-2-vinyl-2,3-dihydro-1,3-benzoxazin-4(4H)-one (31): Pale yellow oil (85.4 mg, 65\%). ${ }^{1} \mathrm{H}$ NMR $\left(400 \mathrm{MHz}, \mathrm{CDCl}_{3}\right): \delta 8.83(\mathrm{~d}, J=2.8 \mathrm{~Hz}, 1 \mathrm{H}), 8.30(\mathrm{dd}, J=8.9,2.8 \mathrm{~Hz}, 1 \mathrm{H}), 7.05(\mathrm{~d}, J=8.9 \mathrm{~Hz}, 1 \mathrm{H})$, $5.94(\mathrm{ddd}, J=17.0,10.3,5.4 \mathrm{~Hz}, 1 \mathrm{H}), 5.78(\mathrm{dd}, J=5.4,0.8 \mathrm{~Hz}, 1 \mathrm{H}), 5.44(\mathrm{dd}, J=17.0,0.8 \mathrm{~Hz}, 1 \mathrm{H}), 5.44(\mathrm{~d}$, $J=10.3 \mathrm{~Hz}, 1 \mathrm{H}), 3.97(\mathrm{ddd}, J=14.0,8.2,6.8 \mathrm{~Hz}, 1 \mathrm{H}), 3.01(\mathrm{ddd}, J=14.0,8.2,6.8 \mathrm{~Hz}, 1 \mathrm{H}), 1.83-1.67$ $(\mathrm{m}, 2 \mathrm{H}), 0.98(\mathrm{t}, J=7.4 \mathrm{~Hz}, 3 \mathrm{H}) .{ }^{13} \mathrm{C}$ NMR $\left(100 \mathrm{MHz}, \mathrm{CDCl}_{3}\right): \delta 159.9,159.5,143.0,131.6,129.2,124.5$, $121.9,118.8,118.0,88.2,46.2,21.5,11.4$. HRMS (ESI) $m / z$ : $[\mathrm{M}+\mathrm{H}]^{+}$calcd for $\mathrm{C}_{13} \mathrm{H}_{15} \mathrm{~N}_{2} \mathrm{O}_{4}, 263.1026$; found, 263.1023.

6-Trifluoromethyl-N-propyl-2-vinyl-2,3-dihydro-1,3-benzoxazin-4(4H)-one (3m): Pale yellow oil (103.8 mg, 73\%). ${ }^{1} \mathrm{H}$ NMR $\left(400 \mathrm{MHz}, \mathrm{CDCl}_{3}\right): \delta 8.22(\mathrm{~d}, J=2.1 \mathrm{~Hz}, 1 \mathrm{H}), 7.63(\mathrm{dd}, J=8.6,2.1 \mathrm{~Hz}, 1 \mathrm{H}), 7.01(\mathrm{~d}$, $J=8.6 \mathrm{~Hz}, 1 \mathrm{H}), 5.94(\mathrm{ddd}, J=16.4,10.4,5.5 \mathrm{~Hz}, 1 \mathrm{H}), 5.71(\mathrm{~d}, J=5.5 \mathrm{~Hz}, 1 \mathrm{H}), 5.40(\mathrm{~d}, J=16.4 \mathrm{~Hz}$, $1 \mathrm{H}), 5.40(\mathrm{~d}, J=10.4 \mathrm{~Hz}, 1 \mathrm{H}), 3.95(\mathrm{ddd}, J=14.1,8.0,6.5 \mathrm{~Hz}, 1 \mathrm{H}), 2.98(\mathrm{ddd}, J=14.1,8.0,6.5 \mathrm{~Hz}, 1 \mathrm{H})$, 1.72-1.62 (m, 2H), $0.97(\mathrm{t}, J=7.4 \mathrm{~Hz}, 3 \mathrm{H}) .{ }^{13} \mathrm{C}$ NMR $\left(100 \mathrm{MHz}, \mathrm{CDCl}_{3}\right): \delta 160.2,157.7,132.0,130.84(\mathrm{q}$, $J=13.0 \mathrm{~Hz}), 125.8(\mathrm{q}, J=13.0 \mathrm{~Hz}), 124.9(\mathrm{q}, J=34.0 \mathrm{~Hz}), 123.9(\mathrm{q}, J=270.0 \mathrm{~Hz}), 121.5,118.7,117.6$, 87.90, 46.02, 21.55, 11.36. HRMS (ESI) $\mathrm{m} / z$ : $[\mathrm{M}+\mathrm{H}]^{+}$calcd for $\mathrm{C}_{14} \mathrm{H}_{15} \mathrm{~F}_{3} \mathrm{NO}_{2}, 286.1049$; found, 286.1046 . 


\section{Conclusions}

In summary, a facile and efficient solvent-controlled chemodivergent synthesis of 1,4-benzoxazepin-5(4H)-ones and 1,3-benzoxazin-4(4H)-ones via the $\mathrm{KOH}$-promoted cyclization of ortho-fluorobenzamides with 2-propyn-1-ol was developed. The cyclization reaction was proposed to involve the $\mathrm{S}_{\mathrm{N}} \mathrm{Ar}$ reaction of $\mathrm{C}-\mathrm{F}$ bond with 2-propyn-1-ol to give ortho-[(2-propynyl)oxy]benzamide intermediates, which underwent the intramolecular either 7-exo-dig cyclization in a superbase medium of $\mathrm{KOH} / \mathrm{DMSO}$ or a Michael addition of $\mathrm{N}-\mathrm{H}$ to allenyl intermediate in $\mathrm{KOH} / \mathrm{MeCN}$ medium to give different benzo-fused cyclic compounds. The present protocol had the significant advantages of high atom-utilization and high selectivity of product output controlled by simple changing the solvents.

Supplementary Materials: The following are available online: Synthesis of known compound 1, copies of ${ }^{1} \mathrm{H}$ NMR spectra of the prepared 1, copies of NMR spectra of 2, copies of NMR spectra of 3, X-ray structural details of $\mathbf{2 g}$ and $\mathbf{3 g}$, results from the reactions of $\mathbf{1 a}$ with propargyl alcohol in either KOD/D $2 \mathrm{O} / \mathrm{DMSO}$ or KOD/D $2 \mathrm{O} / \mathrm{MeCN}$.

Author Contributions: Investigation, writing-original draft preparation, Q.C.; investigation, Y.W.; conceptualization, supervision, writing-review and editing, R.H.

Funding: Financial support from the National Natural Science Foundation of China (No. 21473097 and No. 21673124).

Conflicts of Interest: The authors declare no conflict of interest.

\section{References and Notes}

1. Wei, Y.; Shi, M. Divergent synthesis of carbo- and heterocycles via gold-catalyzed reactions. ACS Catal. 2016, 6, 2515-2524. [CrossRef]

2. Zhan, G.; Du, W.; Chen, Y.-C. Switchable divergent asymmetric synthesis via organocatalysis. Chem. Soc. Rev. 2017, 46, 1675-1692. [CrossRef] [PubMed]

3. Li, L.; Chen, Z.; Zhang, X.; Jia, Y. Divergent strategy in natural product total synthesis. Chem. Rev. 2018, 118, 3752-3832. [CrossRef] [PubMed]

4. Lee, Y.-C.; Kumar, K.; Waldmann, H. Ligand-directed divergent synthesis of carbo- and heterocyclic ring systems. Angew. Chem. Int. Ed. 2018, 57, 5212-5226. [CrossRef] [PubMed]

5. Xu, Z.; Wang, Q.; Zhu, J. Metamorphosis of cycloalkenes for the divergent total synthesis of polycyclic indole alkaloids. Chem. Soc. Rev. 2018, 47, 7882-7898. [CrossRef]

6. Ding, D.; Mou, T.; Xue, J.; Jiang, X. Access to divergent benzo-heterocycles via a catalyst-dependent strategy in the controllable cyclization of o-alkynyl-N-methoxyl-benzamides. Chem. Commun. 2017, 53, 5279-5282. [CrossRef]

7. Thenarukandiyil, R.; Dutta, C.; Choudhury, J. Switching of reaction pathway from C-C rollover to C-N ring-extension annulations. Chem. Eur. J. 2017, 23, 15529-15533. [CrossRef]

8. Gao, W.-C.; Liu, T.; Cheng, Y.-F.; Chang, H.-H.; Li, X.; Zhou, R.; Wei, W.-L.; Qiao, Y. $\mathrm{AlCl}_{3}$-catalyzed intramolecular cyclization of $\mathrm{N}$-arylpropynamides with $\mathrm{N}$-sulfanylsuccinimides: Divergent synthesis of 3-sulfenyl quinolin-2-ones and azaspiro [4,5]trienones. J. Org. Chem. 2017, 82, 13459-13467. [CrossRef]

9. Xu, Y.; Zheng, G.; Yang, X.; Li, X. Rhodium(III)-catalyzed chemodivergent annulations between $\mathrm{N}$-methoxybenzamides and sulfoxonium ylides via C-H activation. Chem. Commun. 2018, 54, 670-673. [CrossRef]

10. Sun, J.; Bai, D.; Wang, P.; Wang, K.; Zheng, G.; Li, X. Chemodivergent oxidative annulation of benzamides and enynes via 1,4-Rhodium migration. Org. Lett. 2019, 21, 1789-1793. [CrossRef]

11. Davies, D.L.; Ellul, C.E.; Macgregor, S.A.; McMullin, C.L.; Singh, K. Experimental and DFT studies explain solvent control of $\mathrm{C}-\mathrm{H}$ activation and product selectivity in the $\mathrm{Rh}$ (III)-catalyzed formation of neutral and cationic heterocycles. J. Am. Chem. Soc. 2015, 137, 9659-9669. [CrossRef] [PubMed]

12. Wang, H.; Lu, Q.; Qian, C.; Liu, C.; Liu, W.; Chen, K.; Lei, A. Solvent-enabled radical selectivities: Controlled syntheses of sulfoxides and sulfides. Angew. Chem. Int. Ed. 2016, 55, 1094-1097. [CrossRef] [PubMed]

13. Xie, T.; Xiao, Y.; Zhao, S.; Hu, X.-Q.; Xu, P.-F. Catalyst-free chemoselective synthesis of 3,4-dihydroquinazoline-2-thiones and 2-imino[1,3]benzothiazines. J. Org. Chem. 2016, 81, 10499-10505. [CrossRef] [PubMed] 
14. Hu, W.; Li, Z.; Li, J.; Wu, W.; Liu, H.; Jiang, H. Palladium-catalyzed cross-coupling of alkynyl carboxylic acids with isocyanides: Solvent-controlled selective synthesis of 5-iminofuranones and 5-iminopyrrolones. Adv. Synth. Catal. 2017, 359, 3509-3514. [CrossRef]

15. Jia, J.; Yu, A.; Ma, S.; Zhang, Y.; Li, K.; Meng, X. Solvent-controlled switchable domino reactions of MBH carbonate: Synthesis of benzothiophene fused $\alpha$-pyran, 2,3-dihydrooxepine and oxatricyclodecene derivatives. Org. Lett. 2017, 19, 6084-6087. [CrossRef]

16. Peng, J.-B.; Wu, X.-F. Ligand- and solvent-controlled regio- and chemodivergent carbonylative reactions. Angew. Chem. Int. Ed. 2018, 57, 1152-1160. [CrossRef]

17. Guo, S.; Wang, F.; Tao, L.; Zhang, X.; Fan, X. Solvent-dependent copper-catalyzed indolyl C3-oxygenation and N1-cyclization reactions: Selective synthesis of 3H-indol-3-ones and indolo[1,2-c]quinazolines. J. Org. Chem. 2018, 83, 3889-3896. [CrossRef]

18. Zhang, H.-H.; Wang, Y.-Q.; Huang, L.-T.; Zhu, L.-Q.; Feng, Y.-Y.; Lu, Y.-M.; Zhao, Q.-Y.; Wang, X.-Q.; Wang, Z. NaI-mediated divergent synthesis of isatins and isoindigoes: A new protocol enabled by an oxidation relay strategy. Chem. Commun. 2018, 54, 8265-8268. [CrossRef]

19. Wu, F.-P.; Peng, J.-B.; Qi, X.; Ying, J.; Wu, X.-F. Palladium-catalyzed solvent-dependent divergent synthesis of benzylformamides. Adv. Synth. Catal. 2018, 360, 3412-3417. [CrossRef]

20. Yi, W.; Chen, W.; Liu, F.-X.; Zhong, Y.; Wu, D.; Zhou, Z.; Gao, H. Rh(III)-catalyzed and solvent-controlled chemoselective synthesis of chalcone and benzofuran frameworks via synergistic dual directing groups enabled regioselective $\mathrm{C}-\mathrm{H}$ functionalization: A combined experimental and computational study. ACS Catal. 2018, 8, 9508-9519. [CrossRef]

21. Xu, L.; Chen, J.; Chu, L. Solvent-tuned chemoselective carboazidation and diazidation of alkenes via iron catalysis. Org. Chem. Front. 2019, 6, 512-516. [CrossRef]

22. Kamei, K.; Maeda, N.; Ogino, R.; Koyama, M.; Nakajima, M.; Tatsuoka, T.; Ohno, T.; Inoue, T. New 5-HT 1 A receptor agonists possessing 1,4-benzoxazepine scaffold exhibit highly potent anti-ischemic effects. Bioorg. Med. Chem. Lett. 2001, 11, 595-598. [CrossRef]

23. Kamei, K.; Maeda, N.; Nomura, K.; Shibata, M.; Katsuragi-Ogino, R.; Koyama, M.; Nakajima, M.; Inoue, T.; Ohno, T.; Tatsuoka, T. Synthesis, SAR studies, and evaluation of 1,4-benzoxazepine derivatives as selective 5-HT1A receptor agonists with neuroprotective effect: Discovery of Piclozotan. Bioorg. Med. Chem. 2006, 14, 1978-1992. [CrossRef] [PubMed]

24. Deng, X.-Q.; Song, M.-X.; Wei, C.-X.; Sun, Z.-G.; Quan, Z.-S. Synthesis and evaluation of 7-substituted-3,4dihydrobenzo[f]- [1,4]oxazepin-5(2H)-ones as anticonvulsant and hypnotic agents. Med. Chem. Res. 2011, 20, 996-1004. [CrossRef]

25. Liao, J.-Y.; Shao, P.-L.; Zhao, Y. Catalytic divergent synthesis of $3 H$ or $1 H$ pyrroles by [3 +2 ] cyclization of allenoates with activated isocyanides. J. Am. Chem. Soc. 2015, 137, 628-631. [CrossRef]

26. Feng, J.-J.; Lin, T.-Y.; Zhu, C.-Z.; Wang, H.; Wu, H.-H.; Zhang, J. The divergent synthesis of nitrogen heterocycles by rhodium(I)-catalyzed intermolecular cycloadditions of vinyl aziridines and alkynes. J. Am. Chem. Soc. 2016, 138, 2178-2181. [CrossRef]

27. Naganathan, S.; Andersen, D.L.; Andersen, N.G.; Lau, S.; Lohse, A.; Sørensen, M.D. Process development and scale-up of a benzoxazepine-containing kinase inhibitor. Org. Process Res. Dev. 2015, 19, 721-734. [CrossRef]

28. Popp, T.A.; Tallant, C.; Rogers, C.; Fedorov, O.; Brennan, P.E.; Müller, S.; Knapp, S.; Bracher, F. Development of selective CBP/P300 benzoxazepine bromodomain inhibitors. J. Med. Chem. 2016, 59, 8889-8912. [CrossRef]

29. Cheng, Q.-Q.; Lankelma, M.; Wherritt, D.; Arman, H.; Doyle, M.P. Divergent rhodium-catalyzed cyclization reactions of enoldiazoacetamides with nitrosoarenes. J. Am. Chem. Soc. 2017, 139, 9839-9842. [CrossRef]

30. Monzani, M.V.; Coltro, G.; Sala, A.; Sardina, M. Pharmacokinetics of ITF 296 (Sinitrodil) a novel organic nitrate, in healthy volunteers. Eur. J. Pharmaceut. Sci. 1999, 7, 179-184. [CrossRef]

31. Minghetti, P.; Casiraghi, A.; Montanari, L.; Monzani, M.V. In vitro skin permeation of Sinitrodil, a member of a new class of nitrovasodilator drugs. Eur. J. Pharmaceut. Sci. 1999, 7, 231-236. [CrossRef]

32. Madhavan, G.R.; Chakrabarti, R.; Reddy, K.A.; Rajesh, B.M.; Balraju, V.; Rao, P.B.; Rajagopalan, R.; Iqbal, J. Dual PPAR- $\alpha$ and $-\gamma$ activators derived from novel benzoxazinone containing thiazolidinediones having antidiabetic and hypolipidemic potential. Bioorg. Med. Chem. 2006, 14, 584-591. [CrossRef] [PubMed]

33. Chouhan, G.; Alper, H. Domino ring-opening/carboxamidation reactions of $N$-tosyl aziridines and 2-halophenols/pyridinol: Efficient synthesis of 1,4-benzo- and pyrido-oxazepinones. Org. Lett. 2010, 12, 192-195. [CrossRef] 
34. Pandey, S.; Kumar, S.V.; Kant, R.; Chauhan, P.M.S. Base mediated 7-exo-dig intramolecular cyclization of Ugi-propargyl precursors: A highly efficient and regioselective synthetic approach toward diverse 1,4-benzoxazepine-5(2H)-ones. Org. Biomol. Chem. 2014, 12, 5346-5350. [CrossRef]

35. Meiresonne, T.; Verniest, G.; Kimpe, N.D.; Mangelinckx, S. Synthesis of 2-fluoro-1,4-benzoxazines and 2-fluoro-1,4-benzoxazepin-5-ones by exploring the nucleophilic vinylic substitution $\left(\mathrm{S}_{\mathrm{N}} \mathrm{V}\right)$ reaction of gem-difluoroenamides. J. Org. Chem. 2015, 80, 5111-5124. [CrossRef] [PubMed]

36. Su, J.; Chen, Q.; Lu, L.; Ma, Y.; Auyoung, G.H.L.; Hua, R. Base-promoted nucleophilic fluoroarenes substitution of C-F bonds. Tetrahedron 2018, 74, 303-307. [CrossRef]

37. Iqbal, M.A.; Mehmood, H.; Lv, J.; Hua, R. Base-promoted $\mathrm{S}_{\mathrm{N}}$ Ar reactions of fluoro- and chloroarenes as a route to $\mathrm{N}$-aryl indoles and carbazoles. Molecules 2019, 24, 1145. [CrossRef]

38. Iqbal, M.A.; Lu, L.; Mehmood, H.; Khan, D.M.; Hua, R. Quinazolinone synthesis through base-promoted $\mathrm{S}_{\mathrm{N}}$ Ar reaction of ortho-fluorobenzamides with amides followed by cyclization. ACS Omega 2019, 4, 8207-8213. [CrossRef]

39. Huang, Q.; Hua, R. Rhodium(I)-catalyzed reductive cyclocarbonylation of internal alkynes: Atom- economic process for synthesis of 2-cyclopenten-1-ones, 5-alkylidenefuran- 2(5H)-ones and indan-1-ones. Chem. Eur. J. 2009, 15, 3817-3822. [CrossRef]

40. Li, J.; Hua, R. Stereodivergent ruthenium-catalyzed transfer semihydrogenation of diaryl alkynes. Chem. Eur. J. 2011, 17, 8462-8465. [CrossRef]

41. Nizami, T.A.; Hua, R. Silver-catalyzed chemoselective annulation of propargyl amines with alkynes for access to pyridines and pyrroles. Tetrahedron 2017, 73, 6080-6084. [CrossRef]

42. Nizami, T.A.; Hua, R. Cycloaddition of 1,3-butadiynes: Efficient synthesis of carbo- and heterocycles. Molecules 2014, 19, 13788-13802. [CrossRef] [PubMed]

43. Hua, R.; Nizami, T.A. Synthesis of heterocycles by using propargyl compounds as versatile synthons. Mini-Rev. Org. Chem. 2018, 15, 198-207. [CrossRef]

44. Zheng, L.; Hua, R. C-H activation and alkyne annulation via automatic or intrinsic directing groups: Towards high step economy. Chem. Rec. 2018, 18, 556-569. [CrossRef]

45. One substrate example of the cyclization of 2-(2-propynyl)oxy-benzamide under the different base and solvent conditions to afford the corresponding either 3-methyl-1,4-Benzoxazepin-5(4H) -one $(34 \%)$ or 2-vinyl-1,3-Benzoxazin-4(4H)-one (34\%) with low chemoselectivities and low yields was reported, see: Scherrer, V.; Jackson-Mülly, M.; Zsindely, J.; Schmid, H. Base catalysed cyclizations of 2-(2-propynyl)oxy-benzamide systems. Helv. Chim. Acta 1978, 61, 716-731. [CrossRef]

46. Both structures can be confirmed based on their ${ }^{1} \mathrm{H}$ - and ${ }^{13} \mathrm{C}-\mathrm{NMR}$. $2 \mathrm{~g}$ and $\mathbf{3 g}$ are also further confirmed by their X-ray diffraction studies (CCDC1908830 (2g); CCDC1908829 (3g)).

47. Trofimov, B.A.; Schmidt, E.Y. Acetylenes in the superbase-promoted assembly of carbocycles and heterocycles. Acc. Chem. Res. 2018, 51, 1117-1130. [CrossRef]

48. Li, Y.; Chen, J.; Qiu, R.; Wang, X.; Long, J.; Zhu, L.; Au, C.-T.; Xu, X. Cesium hydroxide-catalyzed isomerization of terminal alkynes for the synthesis of $O$-allenes and $N$-allenes. Tetrahedron Lett. 2015, 56, 5504-5507. [CrossRef]

49. As required by one of the reviewers, we have done the D-labeling study by using KOD. Unfortunately, we cannot observe the obvious kinetic isotope effect (KIE), and the experimental results are reported in Supporting Information.

50. Seth, K.; Nautiyal, M.; Purohit, P.; Parikh, N.; Chakraborti, A.K. Palladium catalyzed Csp ${ }^{2}-\mathrm{H}$ activation for direct aryl hydroxylation: The unprecedented role of 1,4-dioxane as a source of hydroxyl radicals. Chem. Commun. 2015, 51, 191-194. [CrossRef]

Sample Availability: Samples of the compounds $\mathbf{2}$ and $\mathbf{3}$ are not available from the authors. 